Referencia para citar este artículo: Montanares-Vargas, E. G. (2017). Estudiantes de Chile y la enseñanza de la historia en espacios de conflicto. Revista Latinoamericana de Ciencias Sociales, Niñez y Juventud, 15 (2), pp. $1237-1250$. DOI:10.11600/1692715x.1523015072016

\title{
Estudiantes de Chile y la enseñanza de la historia en espacios de conflicto*
}

\author{
ELIZABETH GLORIA MoNTANARES-VARGAS \\ Profesora-investigadora Universidad Católica de Temuco, Chile.
}

\section{Artículo recibido en julio 15 de 2016; artículo aceptado en noviembre 28 de 2016 (Eds.)}

- Resumen (descriptivo): en este artículo analizo las creencias de estudiantes de La Araucanía en Chile, sobre aspectos asociados con la enseñanza de la historia de su región. Es un conocimiento que aportaría antecedentes valiosos para incluir en programas del sector; contenidos clave para que el estudiantado comprenda la violencia que se vive hoy en la región estudiada. A partir de la teoría sociocultural del aprendizaje y con una metodología de corte cualitativo-descriptivo, diseñé una entrevista semiestructurada que apliqué a estudiantes secundarios de la región. Con este instrumento identifiqué creencias sobre la historia local y su enseñanza en la escuela. El análisis arrojó resultados como que el estudiantado valora la enseñanza de la historia local, reconociendo que es la familia y no la escuela la fuente principal de conocimiento de la misma.

Palabras clave: enseñanza de la historia, contexto cultural, aprendizaje (Tesauro de Ciencias Sociales de la Unesco).

\section{Chilean students and the teaching of history in conflict zones}

- Abstract (descriptive): this article analyzes the beliefs of secondary students in Araucania, Chile regarding the teaching of the history of their region. This knowledge would contribute valuable content for the programs in this sector so that students could understand the violence that they are currently experiencing in this region. The authors used a socio-cultural learning theory and qualitative-descriptive methodology to design a semi-structured interview guide, an instrument that was applied with secondary students in the region. The analysis produced the conclusion that students value the teaching of local history, recognizing that the main source of knowledge of this history comes from their families and not the school.

Key words: history education, culture, learning. (Social Science Unesco Thesaurus).

\section{Estudantes chilenos e o ensino de história em áreas de conflito}

- Resumo (descritivo): este artigo analisa as crenças dos estudantes secundários de Araucania, no Chile, sobre o ensino da história de sua região. Conhecimento que aportaria antecedentes valiosos para incluir em programas do setor, conteúdos chaves para que o estudante compreenda episódios de violência nos quais vivem hoje os habitantes da região. A partir da teoria da aprendizagem sociocultural e metodologia da parte descritiva qualitativa, uma entrevista semiestruturada foi

Este artículo corto ubicado en el área de las Ciencias Sociales, sub área Educación General, hace parte del Convenio Individual de Desempeño adjudicado a la autora, denominado "Enseñanza de la Historia en Espacios de Conflicto", financiado por la Universidad Católica de Temuco (Contrato de Convenio de Desempeño Individual del 1 de agosto de 2015). Investigación iniciada en el mes de julio de 2015, y que está aún en curso.

** Profesora de Historia, Geografía y Educación Cívica, Magíster en Ciencias Sociales Aplicadas, Universidad de la Frontera, Chile. Docente línea de Didáctica de las Ciencias Sociales y Formación de Profesores Universidad Católica de Temuco, Chile. Investigaciones área Enseñanza de la Historia en espacios de conflicto, Historia Local. Orcid: 0000-0001-9504-2614. Correo electrónico: emontanares@uct.cl 
projetada, instrumento que foi aplicado a estudantes do ensino médio na região de Araucania. A análise encontrou resultados que dizem respeito a como os estudantes valorizam o estudo de sua história local e da educação na escola. A análise e principais resultados mostraram que os alunos valorizam ensino da história local, reconhecendo que é a família não a escola a principal fonte de conhecimento sobre o mesmo.

Palavras chave: ensino de história, contexto cultural, aprendizagem (Thesaurus de Ciências Sociais da Unesco).

\section{-1. Introducción. -2. Creencias, Aprendizaje y Enseñanza de la historia. -3. Enseñanza de la Historia y Conflicto. -4. Estudios sobre la enseñanza de la historia en la Araucanía. -5. Método. -6. Resultados. -7. Discusión y resultados. -Lista de referencias.}

\section{Introducción}

El conflicto mapuche situado en la región de La Araucanía en Chile, sigue afectando hoy a la sociedad regional, con episodios de violencia por parte de supuestos grupos pro mapuche y allanamientos policiales a comunidades indígenas. Esta lucha se ha suscitado por la expulsión que el Estado chileno hizo de las comunidades mapuche para reemplazarlas por colonos, y así desarrollar económicamente la región (Pinto, 2012), con resultados nefastos para la paz social (Llancavil, Sepúlveda, Chacaltana \& Montanares-Vargas, 2015; Pinto, 2012).

Desafortunadamente la escuela, con un currículo históricamente monocultural no entrega herramientas a estudiantes y docentes para leer comprensivamente esta realidad (Mansilla, Llancavil, Mieres \& MontanaresVargas, 2016; Quintriqueo \& Torres, 2013; Williamson \& Hidalgo, 2015).

Una manera de salvar este obstáculo es a través de la enseñanza de la historia, disciplina que puede entregar a la población respuestas despolitizadas (Barton \& Mc Cully, 2012) y herramientas para la construcción de un diálogo intercultural (Quilaqueo, 2013; Quintriqueo \& Torres, 2013) que aprecie opiniones diversas. Se enfocaría la enseñanza en el logro de la paz (Mc Cully \& Clarke, 2016; Smith, Datzberger \& Mc Cully, 2016; Ministerio de Educación, 2014), que es un valor demandado en la zona objeto de estudio. Para ello el profesor o profesora debe mediar, utilizando las percepciones de los estudiantes y las estudiantes -mapuches y no mapuches- sobre la historia local y sus procesos de enseñanza. Estas creencias contextualizarían lo que se enseña en la escuela, permitiendo la construcción de sus propias narrativas históricas traspasadas por el valor que dan a la historia de su región, cuestión central para que se produzca un vínculo entre el pasado y el presente, en una construcción grupal, propiciando discusión y diálogo entre las actuales posiciones contrapuestas.

De esta forma, en este estudio describo las creencias que el estudiantado de la región de la Araucanía posee sobre la historia de la región, su valor y el proceso de enseñanza en el aula, lo que permitirá mejorar las acciones y estrategias de aprendizaje de los alumnos y alumnas en todos los niveles educativos.

Abordo este estudio desde la teoría sociocultural del aprendizaje, es decir, como factor de desarrollo en los individuos, donde el profesor o profesora favorece el desarrollo de las actividades mentales de sus estudiantes, quienes elaboran nuevos conocimientos desde sus propias creencias y estructura cognitiva (Vygotsky, 1986).

\section{Creencias, aprendizaje y enseñanza de la historia}

Distintos estudios abordan la temática sobre creencias en el ámbito de la subjetividad del individuo, unidas a contextos específicos claves en la interpretación que los individuos hacen de su entorno (Kajala \& Barcelos, 2012). Así determinan una forma de estar en el mundo, como filtros mentales frente a las experiencias 
vividas, afectando normas de conducta, decisiones, y percepciones de la realidad que viven los individuos.

La Teoría Sociocultural del Aprendizaje (Vygotsky, 1986) relaciona creencias y aprendizaje, en cuanto a que el conocimiento que reconstruye el alumno o alumna varía según sus experiencias previas, y según el contexto social. Asimismo, estudios recientes sostienen que las creencias docentes son influyentes al momento de educar, en cuanto éstas permiten tomar decisiones fundamentales, como validar y dar forma al conocimiento para enfrentar los procesos de enseñanza y aprendizaje conel objeto de que sus estudiantes puedan acceder a dicho conocimiento (Cortez, Fuentes, Villablanca \& Guzmán, 2013; Guerra \& Balmaceda, 2015; Ormeño \& Rosas, 2015). Cabe señalar que para que se produzca el aprendizaje el profesor o profesora debe conocer las creencias que los sujetos escolares traen sobre las materias para recogerlas y mediar en la construcción de otros nuevos (Pinochet \& Pagés, 2016; Tagle, Díaz, Alarcón, Quintana \& Ramos, 2014).

En definitiva, el sistema de creencias de estudiantes y docentes puede afectar al aprendizaje y las prácticas pedagógicas, interviniendo en la elaboración de aprendizajes nuevos. En el ámbito de la didáctica de la historia, Barton (2010), al sistematizar más de doscientos estudios, concluye que el estudiantado tempranamente tiene conocimiento del pasado, el que es distinto de las expectativas de los educadores y educadoras. Cita estudios de Irlanda del Norte, Canadá y Estados Unidos, que vinculan ideas sobre la historia, el contexto socio cultural, y su efecto en la construcción de nuevos significados (Barton \& Mc Cully, 2010), idea que es confirmada por investigaciones latinoamericanas (Acle \& Bravo, 2013; Ormeño \& Rosas, 2015). Ante este escenario, el profesor o profesora, a partir de las ideas de sus estudiantes, debe iniciar procesos de enseñanza con el fin de mediar en el desarrollo de competencias históricas (Gómez, Ortuño \& Molina, 2014). Al respecto, el uso de fuentes significativas, como fotografías o relatos, podría constituirse en un medio valioso para incentivar interpretaciones contrapuestas entre el estudiantado (Wineburg, Martin \& Monte-Sano, 2013). Ahora, una traba importante que surge, lo son las contradicciones existentes entre aquello que los docentes creen que la historia debe aportar a su alumnado y los medios utilizados para conseguirlo, dentro de los cuales el discurso tradicional es uno de los más utilizados (Tutiax-Guillon, 2003). Una explicación podría estar en el diseño del currículo y los limitados espacios que éste entrega para que en el aula se discutan y validen visiones contrapuestas sobre un mismo hecho histórico. Pero, en definitiva, a pesar de las dificultades, creo que la inclusión de las narrativas y creencias originales del colectivo de estudiantes, más el uso de la metodología histórica en el aula, podrían ser el primer paso en la implementación de un proceso de reconstrucción y resignificación histórica por parte de éstos. En consecuencia, la disciplina histórica aportaría en la construcción de competencias asociadas al diálogo y la comprensión de las visiones distintas que posee el estudiantado de la región, en un contexto complejo, como lo es La Araucanía en Chile.

\section{Enseñanza de la historia y conflicto}

Estudios en el ámbito de la didáctica de la historia en regiones en conflicto valoran su enseñanza para la formación de la identidad individual y comunitaria de la comunidad estudiantil. Especialmente cuando las visiones sobre procesos históricos son fuente de división social, y grupos con experiencias históricas distintas entran en conflicto, entregando visiones neutrales sobre hechos controversiales (Barton \& Mc Cully, 2012; Mc Cully \& Emerson, 2014; Mc Cully \& Clarke, 2016); enseñanza que desarrollaría una identidad integradora de procesos individuales y colectivos, con espacios para todos. Sobre el tema, investigaciones recientes en Chile reconocen el valor de la participación juvenil en la construcción de un currículo aporte en la educación para un futuro mejor (Martínez-Rodríguez, MuñozGrandón \& Muñoz-Labraña, 2016; Pinochet 
\& Pagés, 2016). El éxito de esta tarea depende de reconocer nuevamente que la comprensión histórica y el significado que los estudiantes dan a hechos complejos, son afectados por el origen social y las creencias que éstos poseen (Barton \& Mc Cully, 2012). En ocasiones, son similares a las de los miembros de su comunidad, pero en otras oportunidades coinciden con las que presentan los textos escolares (Levstik \& Barton, 2011; Terzian \& Yeager, 2007). De hecho, hasta podrían ser versiones muy opuestas a las perspectivas oficiales y a las enseñadas en la escuela. Sobre esto, Epstein, Mayorga y Nelson (2011) indican que el origen étnico es tan determinante, que el proceso de evaluación de fuentes primarias podría depender de éste.

Estas diferencias de interpretación de los fenómenos históricos conflictivos, lleva a discutir el rol del docente, el cual debería situar su enseñanza desde múltiples perspectivas históricas abordando también componentes afectivos de lahistoria conflictiva, para promover una reflexión sobre las representaciones contemporáneas del pasado (Barton \& Mc Cully, 2012; Barton, 2015). Al respecto, Mc Cully y Smith (2013) resaltan la labor iniciada por la escuela de educación de la universidad de Ulster hace más de una década, consistente en preparar a los futuros profesores y profesoras para enseñar en espacios que han vivido fuertes conflictos.

La inclusión del ámbito afectivo en programas de historia, para tratar temas difíciles, podría tornarse en una vía para rehacer la conciencia histórica del alumnado desde sus propias necesidades (Cuesta, 2007). En el caso de Chile, estudios sobre el profesorado y la enseñanza de temas conflictivos revelan el silencio que se observa en el aula a propósito de temas traumáticos para el país, como son por ejemplo los relativos a derechos humanos (Magendzo \& Toledo, 2015; Toledo, Magendzo \& Gazmuri, 2011; Toledo, Magendzo, Gutiérrez, Iglesias \& López-Facal, 2015). Este "silencio" ya ha sido tratado en investigaciones más recientes en contextos distintos, como es Irlanda del Norte, arrojando como resultado que el evitar temas controversiales responde a que el profesorado evade aparecer ante los demás politizando la escuela. A esto se suma el temor al desborde de la clase cuando el colectivo de estudiantes manifiesta sus opiniones sobre la sociedad actual. Sin embargo, sí se enseña lo que no es controversial, lo cual prueba que el profesorado sólo soslaya el conflicto con el fin de evitar posibles problemas con autoridades educacionales y apoderados, lo que es más visible en comunidades ubicadas en zonas donde la paz se considera frágil (Håvardstun, 2012; Toledo et al., 2015). Mondaca, Rivera y Aguirre (2013) plantean que el profesorado debe recuperar el papel de actor transformador de la historia, superando la pereza y el miedo para abordar el estudio de la historia reciente desde la óptica descriptiva y mínimamente interpretativa.

En resumen, los estudios vinculados a la enseñanza de la historia en espacios conflictivos, arrojan la necesidad de diseñar un currículo con base en la especificidad de cada localidad, y preparar al cuerpo de docentes desde la formación inicial para que enfrenten cuestiones de tipo emocional. Esto les permitiría asumir la enseñanza de estos temas en comunidades que sufren violencia, ejecutando clases que reconozcan y utilicen las posiciones del estudiantado, promoviendo espacios de diálogo y de paz.

\section{Estudios sobre la enseñanza de la ocupación de La Araucanía}

Los principales estudios asociados a la enseñanza en la región de la Araucanía, vinculan las prácticas pedagógicas a la importancia de los conocimientos que los niños, niñas y jóvenes mapuches poseen como fundamento para la construcción de aprendizajes nuevos. Plantean la necesidad de desarrollar un currículo para la construcción de un diálogo intercultural, que contemple conservar la cultura y la lengua mapuche (Turra-Díaz, Ferrada \& Villena, 2013; Pino \& Merino, 2010; Quintriqueo \& Torres, 2013; Quintriqueo, Quilaqueo, Peña-Cortés \& Muñoz, 2015; Turra-Díaz, 2015). Si bien se han desarrollado investigaciones para superar 
modelos tradicionales de enseñanza, no existen suficientes trabajos referidos especialmente a la enseñanza del proceso de ocupación. Hace una década, Henríquez y Pagés (2004), tras revisar trabajos realizados entre los años 1970 y 2000, plantearon la necesidad de impulsar investigaciones para dialogar con las demandas sociales por las que se veía atravesada la enseñanza y el aprendizaje de la disciplina. Ahora, los estudios coinciden acerca de la urgencia de enmendar errores en la enseñanza de la historia de Chile, la cual evidencia un claro olvido y folcklorización de la comunidad indígena, situación que es visibilizada por los textos escolares (Toledo et al., 2011; Toledo et al., 2015; Villalón \& Pagés, 2015). También las investigaciones enfocadas en la enseñanza de la ocupación, concuerdan en que el currículo prioriza contenidos asociados a la unificación territorial y a la consolidación de fronteras chilenas, provocando que las referencias de comprensión del proceso sean los medios de comunicación, la familia, o los múltiples medios de socialización actual (Rojas, 2010). Esto demuestra que el mismo Estado de Chile no valora este proceso histórico y sus efectos en el clima social de la región, cuestión que se traduce en mantener este currículo disociado de la realidad local.

En este punto, cabe destacar una línea de investigación a partir del análisis de los textos escolares, basada en herramientas mediadoras entre el conocimiento seleccionado en el currículo y la construcción que realiza el estudiantado respecto de este saber (Altamirano, Godoy, Manghi, \& Soto, 2014). Riedemann (2010) recoge los contenidos de los textos de historia, indagando en qué medida éstos ofrecen propuestas interpretativas acerca del vínculo entre la ocupación de la Araucanía y la relación actual entre el Estado y el pueblo mapuche. $\mathrm{Su}$ conclusión es sorprendente, en cuanto tanto el Estado como los mapuche tenderían a describir dicho evento como una serie de sucesos predominantemente pacíficos, y en segundo lugar, omiten el vínculo que existe entre dichos sucesos del pasado y la situación presente de este pueblo.
En su análisis destaca que en dos de los cuatro libros revisados, el contenido y la forma de tratar el conflicto ha sido prácticamente el mismo durante muchos años, y en aquellos textos que fueron modificados, los cambios profundizan en la idea de que la relación entre el Estado chileno y el pueblo mapuche era más bien pacífica, y que la expropiación de tierras no fue algo destacable (Riedemann, 2010). Dichos trabajos entregan una mirada prejuiciada hacia la comunidad mapuche (Villalón \& Pagés, 2015), lo que hace necesario nuevamente revisar el rol del profesorado, que son quienes finalmente deberían actuar en la implementación contextualizada del currículo (Mondaca et al., 2013).

La situación es compleja, puesto que los programas de formación de profesores, tanto de nivel primario como secundario, toman un carácter común o general al sistema escolar, abstrayéndose de los contextos específicos de desempeño, como es el indígena (Turra-Díaz, 2012). Concuerdan con esta afirmación los resultados obtenidos por Segura y Núñez (2011), a partir de una investigación que describe las características de las prácticas de profesores y profesoras de segundo año medio que se desempeñan en el sector de historia y ciencias sociales, en relación con la enseñanza del pueblo mapuche; la enseñanza de las ciencias sociales está enmarcada en un modelo tradicional, lo que trasciende al tipo de establecimiento y al emplazamiento de la comuna.

Más sorprendente resulta el que las profesoras y profesores consultados desconocieron absolutamente el "conflicto" en la Araucanía. Toda la información que poseen, dice relación con el "conflicto territorial" de un grupo de comuneros en un lugar determinado de la región, donde se pelean por un terreno, quemando camiones, cortando bosques $\mathrm{y}$ tomándose terrenos de personas propietarias. Lo anterior se vincula con lo expuesto por varios autores y autoras, en cuanto se apela a la necesidad de integrar al aula contenidos socioculturales mapuche, lo que se dificulta por la escolarización monocultural recibida por los profesores y profesoras de origen 
mapuche y no mapuche (Turra-Díaz et al., 2013; Pino \& Merino, 2010; Quintriqueo \& Torres, 2013; Quintriqueo et al., 2015; TurraDíaz, 2015), postura que es corroborada por el análisis multimodal de un texto escolar de historia que explica que los textos, además de presentar los conceptos a través del lenguaje, utilizan las imágenes, las que, en el caso de los pueblos originarios, corresponden a caricaturas o dibujos simbólicos, imaginarios, presentados sin rigor histórico, sin ser usados como fuentes secundarias ni citar autoría de la representación visual. Esto otorga nuevas preguntas y proyecciones de investigación, como por ejemplo la necesidad de reflexionar si es necesario preparar a los sujetos docentes desde su formación inicial, para trabajar de forma multimodal y desarrollar estrategias al respecto (Altamirano et al., 2014).

Desde otra mirada, Turra-Díaz (2012), en estudio ya citado dirigido a indagar en la definición curricular del sector de aprendizaje Historia y Ciencias Sociales en un contexto indígena intercultural (Provincia de AraucoChile), aborda la narrativa histórica mapuche, sobre la cual señala que hay una ausencia de contenidos específicos en el sector de aprendizaje que den cuenta de la narrativa histórica mapuche en los procesos formativos del estudiantado perteneciente a este pueblo indígena (Turra-Díaz, 2012). Sin embargo, desde otra perspectiva un trabajo más reciente, concluye que la principal fuente de información para los jóvenes de la región de la Araucanía es la escuela y la clase de historia que en ella se imparte, adquiriendo una importancia relevante en las narrativas de los estudiantes, lo cual se refleja en, por ejemplo, la visión que tienen de la historia de este pueblo y del sujeto mapuche (Areyuna, Bruzzone, López \& Pizarro, 2014).

De esa forma, hay claridad en que el currículo chileno no ha asumido la enseñanza de la historia de la Araucanía como una necesidad para la región y para el país. Al contrario, la ocupación es una temática que es abordada sin tener en cuenta el punto de vista mapuche; incluso se lo ha reducido a explicar el proceso de unificación territorial. Lo anterior es coherente con los estudios basados en los textos escolares diseñados por el ministerio de educación, los que denotan una invisibilidad o tergiversación de la problemática mapuche. Desde el punto de vista de los profesores y profesoras, éstos parecen no recibir desde las universidades una formación para enseñar procesos históricos complejos que son fuente de división social.

\section{Método}

Para alcanzar el objetivo de esta investigación, adopté una metodología de carácter cualitativo descriptiva, para acceder al mundo personal, de ideas y creencias de los sujetos, y conocer el valor que el colectivo de estudiantes secundarios de la región de la Araucanía, Chile, le da al estudio de la historia local. Para Pérez-Serrano (1994), esta metodología permite una mejor comprensión del contexto, ayudándonos a situar en el marco en el que ocurre el acontecimiento, permitiéndonos así obtener una visión más global de la realidad, o sea, una atención especial al contexto, circunstancias y ámbitos en los que se desarrolla el comportamiento humano.

Elegí el diseño de estudio de casos, centrado en los rasgos profundos y en las características del objeto de estudio (Mckernan, 2001), desde un abordaje fenomenológico, ya que representa la forma como los sujetos participantes y la investigadora lo experimentamos. Dentro de los estudios de caso elegí específicamente los casos colectivos, lo que quiere decir que de un grupo escogí a varios informantes que en su conjunto representan el problema en cuestión.

\subsection{Participantes}

Utilicé una muestra intencionada en un centro educativo subvencionado y mixto de la ciudad de Freire, comuna de la región de la Araucanía. La población sobre la que hice el estudio está compuesta por estudiantes de segundo año de enseñanza media o secundaria. Participaron 14 individuos jóvenes de un total de 20 matriculados en el curso académico. La edad de los sujetos informantes está entre 
los 15 y 16 años, de los cuales 9 declararon tener origen étnico mapuche y todos afirmaron pertenecer a familias originarias de la región. La selección del establecimiento y del nivel respondió a las condiciones necesarias para desarrollar el estudio: ser un establecimiento educativo de una comuna de la región de la Araucanía, reunir entre su alumnado estudiantes diversos, mapuches y no mapuches, y haber cursado últimamente las materias relativas a la historia reciente de la región, determinadas por el currículo nacional. Las instituciones fueron seleccionadas, previa autorización de directivos y docentes del colegio, y luego procedí a contactar a cada estudiante mediante referencias de los equipos de docentes y de sus propios pares, para luego realizar las entrevistas, previa aceptación del consentimiento informado por parte de los estudiantes y las estudiantes.

\subsection{Recogida de información y análisis de datos}

La técnica de recogida de datos utilizada fue la de entrevistas en profundidad. Esta técnica ha sido descrita como aquella en la que se recolectan datos de los individuos participantes a través de un conjunto de preguntas abiertas formuladas en un orden específico.

Realicé 14 entrevistas, mediante muestra con ayuda de diez preguntas guía que versaban sobre temas relacionados con el origen de los sujetos participantes, la importancia de la clase de historia, la forma en que habían adquirido conocimientos sobre la historia de su región, las estrategias que el profesorado usa para enseñar esta historia $y$, en definitiva, su experiencia personal en relación con el aprendizaje y enseñanza de la historia de la región. Luego del proceso de transcripción de las mismas, para la etapa de análisis seguí el esquema de tareas propuesto por Miles y Huberman (1994). Allí los autores proponen tres fases: la de reducción de los datos, en la que se selecciona la información para hacerla más manejable; la de síntesis y agrupamiento de los datos, y la de verificación de los resultados. Para la primera etapa procedí a separar los segmentos de los discursos de los sujetos informantes que consideré más significativos y que se relacionaban con los objetivos de la investigación. A estos segmentos los denominé "unidades", y el criterio que empleé para su selección fue temático, es decir, identifiqué y seleccioné unidades en función de un tópico. Posteriormente procedí a la categorización de las unidades, es decir, a la clasificación conceptual de las unidades que tenían una idea común. En total establecí tres categorías de análisis: el valor del conocimiento de la historia, expectativas de cada estudiante, fuentes del conocimiento. De éstas se desprendieron las cinco subcategorías: identidad y pertenencia, memoria, conocimiento, demandas territoriales y conflicto mapuche.

Finalizado el análisis de todas las entrevistas, procedí a la interpretación de los datos según lo declarado por los sujetos participantes y conforme a la revisión teórica.

\section{Resultados}

En el análisis de las entrevistas grabadas al grupo de estudiantes, escogí ejemplos paradigmáticos de cada dimensión, con la categoría y subcategorías contenidas.

En la tabla 1 presento los resultados correspondientes a la dimensión que da cuenta de las razones por las cuales cada estudiante cree que es necesario estudiar la historia de la región. Establecí como categoría el valor del conocimiento de la región para cada quien; de ésta se desprenden cuatro subcategorías: identidad y pertenencia, preservación de la memoria, conocimiento y demandas territoriales (Tabla1). 
Tabla 1. Razones por las cuales es necesario estudiar la historia de la región.

\begin{tabular}{|c|c|c|c|}
\hline Dimensión & Categoría & Subcategoría & Alumnos y alumnas \\
\hline $\begin{array}{l}\text { ¿Cuáles son las razones } \\
\text { por las cuales es } \\
\text { necesario estudiar la } \\
\text { Historia de la Región? }\end{array}$ & $\begin{array}{l}\text { Valor del } \\
\text { conocimiento }\end{array}$ & $\begin{array}{l}\text { Identidad y } \\
\text { pertenencia }\end{array}$ & $\begin{array}{l}\text { "Yo creo que si para saber más de dónde venimos, } \\
\text { de dónde nacemos" (E2) } \\
\text { "yo creo que aporta bastante, si alguna vez me } \\
\text { llegan a preguntar algo de eso, puede que lo } \\
\text { sepa puede que no (piensa) pero saber eso de la } \\
\text { Araucanía, si me hacen alguna consulta, sería tonto } \\
\text { que no lo supiera" (E4) } \\
\text { "hay que saber porque si no uno tiene dudas y no } \\
\text { entiende de dónde es"(E 7) } \\
\text { "Sí, es que si uno vive aquí, es necesario saber eso. } \\
\text { Porque si viene gente de otra parte para contarle" } \\
\text { (E1) } \\
\text { "para no olvidar lo que pasó en este lugar hace } \\
\text { años" (E7) } \\
\text { "Así podemos entender el presente de la región, } \\
\text { me ayuda a tener opinión acerca de lo que está } \\
\text { pasando" (E8) } \\
\text { "Saber los métodos que los mapuches tuvieron para } \\
\text { seguir cultivando" (E1) } \\
\text { "Saber y explicar lo que pasa en este lugar a los que } \\
\text { preguntan"(E12) } \\
\text { "Ayuda a entender lo que pasa hoy en día, lo de los } \\
\text { problemas mapuches" (E4) } \\
\text { "para saber lo que pasa hoy con las tierras y esas } \\
\text { cosas de pelea" (E1) } \\
\text { "comprender por qué piden la tierra los } \\
\text { mapuches"(E14) } \\
\text { "Las intenciones, que ahora están haciendo, que } \\
\text { quieran recuperar sus tierras" (E1) }\end{array}$ \\
\hline
\end{tabular}

En la primera subcategoría se hace referencia al hecho de que por vivir en la región es necesario conocer la historia. Llama la atención la asociación entre el conocimiento de la región y el conocimiento personal. De igual forma, resalta el hecho de saber para poder explicar a otros. En la segunda subcategoría se mencionan aspectos relativos a su rol futuro como transmisor de esta historia. Se puede observar la relevancia que se le da a la entrega del conocimiento a otros, a gente de otra parte. La tercera subcategoría muestra la relación entre conocer la historia para saber e investigar, con aspectos puntuales de la historia mapuche, lo que les ayudaría a saber lo que está pasando. Es interesante lo que expresan sobre conocer las formas de producción y la manera de cultivar la tierra de los mapuches.
Luego, la última subcategoría hace directa alusión a la importancia de estudiar la historia para conocer sobre la problemática mapuche, específicamente para comprender la razón de las demandas territoriales y la situación de la región. En la tabla 2 se presentan los resultados de lo declarado por los estudiantes en relación con los aspectos de la historia de la región que desearían profundizar.

Al revisar la segunda dimensión (Tabla 2) que trata sobre los aspectos que las personas informantes quisieran profundizar sobre la historia de la región, se desprende sólo una subcategoría: conflicto mapuche. 
Tabla 2. Aspectos a profundizar de la historia de la región.

\begin{tabular}{|c|c|c|c|}
\hline Dimensión & Categoría & Subcategoría & Alumnos y alumnas \\
\hline $\begin{array}{l}\text { ¿Qué aspectos de la historia } \\
\text { de la región te interesaría } \\
\text { profundizar? }\end{array}$ & $\begin{array}{l}\text { Expectativas } \\
\text { estudiantes }\end{array}$ & $\begin{array}{l}\text { Conflicto } \\
\text { mapuche }\end{array}$ & $\begin{array}{l}\text { "Me hubiera gustado ver así más completo } \\
\text { así, detallado el conflicto que tenían los } \\
\text { mapuches y los españoles" (E2) } \\
\text { "La lucha de los mapuche" (E3) } \\
\text { "El problema de las tierras que se quitaron } \\
\text { a los mapuches y ahora las están pidiendo" } \\
\text { (E8) } \\
\text { "Podríamos haber visto una película sobre lo } \\
\text { de las tierras y peleas de acá" (E 5) } \\
\text { "También que otros vengan a explicarnos } \\
\text { acá sobre el problema de las peleas que hoy } \\
\text { existen" (E 13) }\end{array}$ \\
\hline
\end{tabular}

Se aprecia que los sujetos participantes quisieran más detalle sobre conflictos pasados y actuales, tanto entre mapuches y españoles, como entre mapuches y chilenos, en la lucha que el pueblo mapuche sostiene. También hay evidencia del interés de conocer más sobre el problema de las tierras y las razones por las cuales se las quitaron a los mapuches. De igual forma, las respuestas denotan un grado importante de identificación, por parte de los individuos entrevistados, con el problema que está viviendo el pueblo mapuche; no así sobre situaciones que afectan a la población no mapuche.
También se observa a través de las textualidades, las escasas respuestas que la escuela entrega mediante los contenidos determinados por el currículo, sobre la historia local de la región de la Araucanía. Las inquietudes que se observan en las respuestas denotan desconocimiento sobre las razones de la violencia existente en la región, pero preocupación por ésta.

De la tercera dimensión presentada en la tabla 3, referente a las fuentes de adquisición del conocimiento, se desprenden dos subcategorías: familia y escuela.

Tabla 3: Fuentes de conocimiento.

\begin{tabular}{|l|l|l|l|}
\hline \multicolumn{1}{|c|}{ Dimensión } & Categoría & Subcategoría & \multicolumn{1}{c|}{ Alumnos y alumnas } \\
\hline $\begin{array}{l}\text { ¿Cuáles han sido las fuentes } \\
\text { desde donde has adquirido el } \\
\begin{array}{l}\text { conocimiento de la Historia de la } \\
\text { Región? }\end{array}\end{array}$ & $\begin{array}{l}\text { Fuentes } \\
\text { conocimiento }\end{array}$ & $\begin{array}{l}\text { Familia } \\
\text { "Mi tía está en una comunidad y sabe } \\
\text { much ella de repente va a la casa y dice } \\
\text { me intereso" (E1) } \\
\text { "No mucho, mi abuelo, mis tíos, mi mamá } \\
\text { me cuenta las historias de sus abuelos, mi } \\
\text { abuelo de su papá, de cómo le quitaron las } \\
\text { tierras" (E5) }\end{array}$ \\
\hline & $\begin{array}{l}\text { "El profesor de historia que nos enseña" } \\
\text { (E2) } \\
\text { "Del colegio de las clases de historia" (E4) }\end{array}$ \\
\hline
\end{tabular}

Las textualidades muestran la importancia del entorno familiar de los sujetos entrevistados en la adquisición de conocimiento sobre la historia local. Los saberes están asociados a narraciones de familiares directos como tías, abuelos, madres y padres. La pertenencia a la comunidad como factor que facilita el acceso a conocimiento sobre el pasado común, que es un factor a tener en cuenta. Igualmente se observa que es un conocimiento escaso, al cual acceden en forma intermitente. El rol de la escuela es descrito como menor en relación con la familia; 
incluso se indica que sólo en una oportunidad o en forma muy superficial es fuente para aprender de historia de la región.

\section{Discusión y conclusiones}

En este artículo reviso evidencia empírica existente sobre creencias de estudiantes, estudios sobre espacios de conflicto y enseñanza de la historia, y examino las creencias de diez estudiantes de enseñanza secundaria de la Araucanía, Chile, en relación con la importancia de estudiar la historia de la región. Mi punto de partida en este artículo es la necesidad de conocer las creencias de los estudiantes y las estudiantes de la Araucanía, sobre el valor que dan a la enseñanza de la historia de la región, con el fin de propiciar una enseñanza que les permita a los estudiantes construir "narrativas históricas" propias a partir de las cuales logren una mayor comprensión de la historia de su región y de las situaciones de conflicto que ocurren. Al revisar la literatura, ya en 2010, Barton sistematiza alrededor de 200 estudios empíricos que están centrados en las ideas de los estudiantes y la historia.

En el presente estudio constaté que el estudiantado de la región valora el estudio de la historia de la Araucanía, y vincula su aprendizaje con los problemas mapuches y la violencia del pasado y del presente. La necesidad, según lo expresa el colectivo de estudiantes, está en obtener conocimiento para fortalecer su propia identidad, para transmitir el conocimiento a otros y para obtener respuestas sobre el conflicto indígena del pasado y sobre el que se vive en la actualidad. Reconocen que el conocimiento de la historia del lugar que habitan es un aporte al conocimiento de sí mismos y a la comprensión de la realidad en la que viven. De esa forma he podido comprobar que la historia juega un rol importante en la formación de la identidad individual y comunitaria de los niños, niñas y jóvenes, cuando grupos con experiencias históricas distintas entran en conflicto (Barton, 2015).

Igualmente los individuos entrevistados mostraron una clara inquietud de profundizar sobre el conflicto mapuche y la razón por la cual les quitaron las tierras. Así he corroborado resultados de investigaciones anteriores que señalan que la educación histórica es una herramienta que le permite al sujeto "lidiar con el pasado reciente", aportar en la reconciliación en lugares que han vivido largos conflictos (Mc Cully \& Clarke, 2016). Cabe señalar que, aun cuando nunca fue mencionado por las personas entrevistadoras, la preocupación por comprender el conflicto fue un punto de coincidencia entre los individuos entrevistados, y surgió naturalmente como motivación central para estudiar la historia local.

Asimismo, se reconoce a la familia como la principal fuente de conocimiento de la historia de la ocupación, con una muy breve alusión del estudiantado al rol del profesor de historia, lo que contrasta con lo indicado por Areyuna et al. (2014), sobre que las principales fuentes de conocimiento en la región están en la escuela. Se estima que la temática relacionada con la ocupación de la Araucanía, es abordada pobremente por los planes y programas chilenos, tanto por la brevedad de los contenidos seleccionados, como porque el enfoque temático escogido dificulta cualquier intento de vinculación con los problemas actuales de la región.

Lo anterior es congruente con investigaciones similares consultadas que dieron cuenta de la incoherencia entre las materias propuestas por el ministerio y el silencio que se observa en el currículo chileno sobre temas traumáticos (Toledo et al., 2011). Otros estudios confirman este análisis crítico de la información oficial entregada por el currículo, a propósito de la guerra del Pacífico (Mondaca et al., 2013). También la revisión de investigaciones sobre los textos escolares y el tratamiento que éstos hacen de los contenidos relativos a la ocupación, iluminan el poco protagonismo que el profesor o profesora parece tener en la adquisición de conocimiento de sus estudiantes. Más grave aún es que los textos entregan una mirada hegemónica y discriminatoria, incluso con imágenes distorsionadas de los pueblos originarios, en ausencia del debido rigor histórico (Altamirano et al., 2014; Villalón \& Pagés, 2015), imágenes que tenderían a describir eventos pacíficos y 
totalmente desvinculados de lo que hoy vive el pueblo mapuche (Riedemann, 2010).

$\mathrm{Si}$ bien el material de enseñanza condiciona en gran medida la labor que el profesorado de historia puede realizar en el aula, cabe preguntarse ¿cuál es la capacidad del sujeto docente de leer el contexto y acercar los contenidos a la realidad del estudiantado? En ese aspecto, los trabajos de Turra-Díaz (2012), y de Segura y Núñez (2011), declaran debilidades importantes en los programas de formación de docentes que se abstraen de los contextos específicos de desempeño, llegando a desconocer la existencia de un conflicto en la región de la Araucanía (Segura \& Núñez, 2011; Turra-Díaz, 2015). Otros estudios incluso señalan que docentes mapuches y no mapuches de la región reciben una escolarización monocultural, lo que limitaría la formación de docentes y estudiantes de la región (Quintriqueo \& Torres, 2012; Turra-Díaz, 2015).

Ahora, sobre las implicaciones prácticas del estudio, éstas dicen relación con las políticas públicas y la necesidad de discutir el diseño de un currículo pertinente al contexto sociocultural de la región, cuestión que se ha venido discutiendo en estudios internacionales, nacionales y locales (Barton, 2010; Turra-Díaz, 2012). Luego en el ámbito de la formación de profesores y profesoras, sería importante incluir como una línea de trabajo la enseñanza de la historia en contextos de conflicto. Sin embargo, esto podría quedarse en lo teórico si no se profundiza en el valor y en el rol que la disciplina histórica tiene para la formación de las personas, en ámbitos como la ciudadanía y el logro de la paz, concretamente en la región de la Araucanía en Chile.

También se comprueba la urgente necesidad de promover y enseñar durante la formación inicial docente el uso de fuentes primarias como el método más importante en la adquisición de las herramientas centrales para enseñar en el aula, ya que facilita la adquisición de pensamiento histórico y con este la comprensión de sucesos conflictivos de los cuales los estudiantes y las estudiantes de la región son parte. En ese sentido, es igualmente valioso incentivar un trabajo conjunto entre universidades y docentes en ejercicio, con el fin de fortalecer su trabajo en el aula y comprometerlos con el uso del método histórico, para los fines enunciados.

Por último también como implicaciones prácticas creo que se debe relevar el rescate de la memoria individual y familiar del estudiantado, como fuente de conocimiento para el profesor o profesora, con el fin de diseñar actividades que contemplen este aspecto en el proceso de enseñanza/aprendizaje, particularmente en lugares en los que la historia ha sido fuente de divisiones. Asimismo, el recurrir a la memoria permitirá una mayor vinculación entre las prácticas de enseñanza y las vivencias o problemáticas surgidas a partir del contexto sociocultural del colectivo de estudiantes.

\section{Lista de referencias}

Acle, G. \& Bravo, F. (2013). Posturas epistémicas de la historia en estudiantes de la licenciatura en educación primaria. Un estudio de caso. Revista Perfiles Educativos, 35 (42), pp. 87-107.

Altamirano, P.; Godoy, G.; Manghi, D. \& Soto, G. (2014). Analizando los textos de Historia, Geografía y Ciencias Sociales: la configuración multimodal de los pueblos originarios. Revista Estudios Pedagógicos, 1, pp. 263-280. Recuperado de: http://www.scielo.cl/scielo.php?script= sci arttextypid $=\mathrm{S} 07180705201400010$ $00 \overline{1} 6 y \operatorname{lng}=$ esytlng=es. 10.4067/S071807052014000100016

Areyuna, B.; Bruzzone, B.; López, X. \& Pizarro, N. (2014). Conciencia histórica de los estudiantes secundarios sobre la historia del pueblo mapuche. Recuperado de: http:// bibliotecadigital.academia.cl/bitstream/ handle/123456789/3218/TPHIS\%20133. pdf?sequence $=1$ yisAllowed $=y$

Barton, K. (2010). Investigación sobre las ideas de los estudiantes acerca de la historia. Enseñanza de las ciencias sociales: revista de investigación, (9), pp. 97-113.

Barton, K. (2015). Young adolescents' positioning of human rights: findings from Colombia, Northern Ireland, Republic of Ireland and the United States. Research in Comparative and International Education, 10 (1), pp. 48-70. Doi: 
$10.1177 / 1745499914567819$.

Barton, K. \& McCully, A. (2010). You can form your own point of view: internally persuasive discourse in Nothern Ireland Students Encounter s with history. The Teachers College record Journal, 112 (1), pp. 142-181.

Barton, K. \& Mc Cully, A. (2012). Trying to "see things differently: northern Ireland Students' Struggle to Understand Alternative Historical Perspectives. Theory \& Research in Social Education, 40 (4), p. 371-408.

Cortez, K.; Fuentes, V.; Villablanca, I. \& Guzmán, C. (2013). Creencias docentes de profesores ejemplares y su incidencia en las prácticas pedagógicas. Estudios Pedagógicos [online], 39 (2). Recuperado de: $\quad<$ http://mingaonline.uach.cl/scielo. php? script $=$ sci arttext\&ypid $=$ S071807052013000200007\&ylng=es\&ynrm $=\mathrm{i}$ so $>$. ISSN 0718-0705

Cuesta, R. (2007). Los deberes de la memoria en la educación. Barcelona: Octaedro.

Epstein, T.; Mayorga, E. \& Nelson, J. (2011). Teaching about race in and urban history class: the effects of culturally responsive. Teaching. Journal of Social Studies Research, 35 (2), pp. 2-21.

Flick, U. (2012). Introducción a la investigación cualitativa. Madrid: Morata.

Guerra, P. \& Balmaceda, S. (2015). Creencias epistemológicas en profesores que postulan al Programa de Acreditación de Excelencia Pedagógica: análisis descriptivos y comparativos entre profesores que se desempeñan en los diferentes niveles de enseñanza. Estudios Pedagógicos, 41 (2), pp. 107-125. Recuperado de: https://dx.doi. org/10.4067/S0718-07052015000200007

Gómez, C.; Ortuño, J. \& Molina, S. (2014). Aprender a pensar históricamente. Retos para la historia en el siglo XXI. Tempo e Argumento, 6, pp. 5-27.

Håvardstun, S. M. (2012). History education in Northern Ireland: teaching the Northern Ireland conflict in Northern Irish School. Recuperado de: http://urn.nb.no/ URN:NBN:no-32164.

Henríquez, R. \& Pagés, J. (2004). La investigación en didáctica de la historia. Educación XX1, (7), pp. 63-83.

Levstik, L. \& Barton, K. (2011). It wasn`t a good part of history: National Identity and ambiguity in students 'Explanations of historical significance. The Teachers College Records, 99 (3), pp. 478-513.

Llancavil, D. L.; Sepúlveda, J. M.; Chacaltana, M. M. \& Montanares-Vargas, E. G. (2015). La función reproductora de la escuela en la Araucanía, 1883-1910. Revista Austral Ciencias Sociales, 28, pp. 117-135.

Mackernan, J. (2001). Investigación y currículum. Madrid: Morata.

Magendzo, A. \& Toledo, I. (2015). Educación en derechos humanos. Estrategia pedagógica-didáctica centrada en la controversia. Revista Electrónica Educare, 19 (3), pp. 1-16. Recuperado de: https:// doi.org/10.15359/ree.19-3.2.

Mansilla, J.; Llancavil, D.; Mieres, M. \& Montanares-Vargas, E. G. (2016). The monocultural school in Araucanía, 18831910: power devices and Mapuche society. Educação e Pesquisa, 42 (1), pp. 213-228.

Martínez-Rodríguez, R.; Muñoz-Grandón, C. \& Muñoz-Labraña, C. (2016). Percepciones del estudiantado sobre la política, los partidos políticos y las personas dedicadas a la política al finalizar la educación secundaria en Chile. Revista Electrónica Educare, 20, pp. 1-16. Recuperado de: $\quad$ http://www.redalyc.org/articulo. oa? id=194143011017

Mc Cully, A. \& Clarke, L. (2016). A place for fundamental (British) values in teacher education in Northern Ireland? Journal of Education for Teaching, 42 (3), pp. 354368. Recuperado de: http://dx.doi.org/10.1 080/02607476.2016.1184465

Mc Cully, A. \& Emerson, L. (2014). Teaching controversial issues in Northern Ireland. En T. Misco \& J. de Groof (eds.) Crosscultural Case-studies in Controversial Issues: pathways and challenges in democratic citizenship education, (pp. 11274). Tilberg: Legal Wolf Publishers.

Mc Cully, A. \& Smith, R. (2013). Organisational self-evaluation and teacher education for community relations in a transforming 
society? European Journal of Teacher, 36 (3), pp. 320-345.

Miles, M. \& Huberman, M. (1994). Qualitative Data Analysis. Thousand Oaks: Sage.

Ministerio de Educación (2014). Planes y Programas para $7^{\circ}$ y $8^{\circ}$ básico, I, II, III y IV medio. Santiago de Chile: Mineduc.

Mondaca, C., Rivera, O. \& Aguirre C. (2013). La escuela y la guerra del pacífico: propuesta didáctica de historia para la inclusión educativa en contextos transfronterizos del norte de chile". Revista Sí Somos Americanos, 13 (1), pp. 123148. Recuperado de: http://www.scielo. cl/scielo.php?script $=$ sci_arttextypid $=$ S07 1909482013000100006 ylng=esytlng=es. 10.4067/S0719-09482013000100006

Ormeño, V. \& Rosas, M. (2015). Creencias acerca del aprendizaje de una lengua extranjera en un programa de formación inicial de profesores de inglés en Chile. Colomb. Appl. Linguistic. J., 17 (2), pp. 207-228. Doi: http://dx.doi.org/10.14483/ udistrital.jour.calj.2015.2.a03.

Pérez-Serrano, G. (1994). Investigación cualitativa. Retos e interrogantes. I. Métodos. Madrid: La Muralla.

Pino, A. \& Merino, E. (2010). Discriminación e identidad étnica en el discurso oral de adolescentes mapuches en contexto escolar de la ciudad de Temuco. Discurso y Sociedad, 4 (1), pp. 103-119.

Pinochet, S. \& Pagés, J. (2016). ¿Cuál es el protagonismo de los niños, niñas y jóvenes en el currículo de Historia, Geografía y Ciencias Sociales de Chile? El impacto del currículo en las ideas de los niños y jóvenes y su protagonismo en la historia. Praxis Educativa 11 (2), pp. 374, 393.

Pinto, J. (2012). El conflicto Estado-Pueblo Mapuche, 1900-1960. Revista Universum, 27 (1), pp. 167-189. Recuperado de: http://www.scielo.cl/scielo.php?script= sci arttextypid $=$ S071823762012000100 009 ylng=esytlng=es.10.4067/S071823762012000100009

Quilaqueo, D. (2013). Les savoirs éducatifs mapuches. Des défis pour les enseignants En G. Maheux \& R. Gauthier (eds.) La formation des enseignants inuit et des premières nations. Problématiques et pistes d'action, (pp. 107-124). Montréal: Université du Québec.

Quintriqueo, S.; Quilaqueo, R. D.; Peña-Cortés, F. \& Muñoz, T. G. (2015). Conocimientos culturales como contenidos de la educación familiar mapuche. Revista Alpha, (40), pp. 131-146.

Quintriqueo, S. \& Torres, H. (2012). Distancia entre el conocimiento mapuche y el conocimiento escolar en contexto mapuche. Revista Electrónica de Investigación Educativa, 14 (1), pp. 16-33.

Quintriqueo, S. \& Torres, H. (2013). Construcción de conocimiento mapuche $\mathrm{y} \mathrm{su}$ relación con el conocimiento escolar. Estudios Pedagógicos, 39 (1), pp. 199-216. Recuperado de: http:// www.scielo.cl/scielo.php?pid=S0718$07052013000100012 \mathrm{yscript}=$ sci arttext

Riedemann, A. (2010). Textos escolares y conciencia histórica publicada sobre la ocupación de la Araucanía. Santiago de Chile: Mineduc.

Rojas, T. (2010). Temas pendientes en la enseñanza sobre pueblos originarios: una mirada al currículo de Ciencias Sociales. Cuadernos de Educación, (28), pp. 1-3.

Segura, L. \& Núñez, L. (2011). El pueblo Mapuche en el siglo XXI: desafíos del currículum nacional. Concepción: Universidad del Bío Bio.

Smith, A.; Datzberger, S. \& McCully, A. (2016). The Integration of Education and Peacebuilding: Synthesis Report on Myanmar, Pakistan, South Africa and Uganda. New York: Unicef.

Tagle, T.; Díaz, C.; Alarcón, P.; Quintana, M. \& Ramos, L. (2014). Creencias de estudiantes de pedagogía sobre la enseñanza del inglés. Folios, 39, pp. 77-87.

Terzian, S. \& Yeager, E. (2007). That's When We Became a Nation. Urban Latino Adolescents and the Designation of Historical Significance. Urban Education, 42 (1), pp.52-81.

Toledo, M.; Magendzo, A. \& Gazmuri, R. (2011). Teaching recent history in countries that have experienced human rights violations: case studies from Chile. 
Perspectives in Education, 29 (2), pp. 1927.

Toledo, M.; Magendzo, A.; Gutiérrez, V.; Iglesias, R. \& López-Facal, R. (2015). The Teaching of "Controversial Issues" Throughout History, from the Perspective of Chilean Students. Revista de Estudios Sociales (52), pp. 119-133. Doi: http:// dx.doi.org/10.7440/res52.2015.08.

Turra-Díaz, O. (2012). Currículo y construcción de identidad en contextos indígenas chilenos. Educación y Educadores, 15 (1), pp. 81-95. Recuperado de: http://www. redalyc.org/articulo.oa? id $=83424040006$

Turra-Díaz, O. (2015). Profesorado y saberes histórico-educativos mapuche en la enseñanza de la historia. Revista Educare, 19 (3), pp. 3-20. Doi.http//dx.doi. org/1015359/ree.1-3.15.

Turra-Díaz, O.; Ferrada, D. \& Villena, A. (2013). La especificidad del contexto indígena como requerimiento para la formación inicial del profesorado. Estudios Pedagógicos, 39 (2), pp. 329-339.

Tutiax-Guillon, N. (2003). Los fundamentos de una investigación sobre la concepción de las finalidades cívicas y culturales del profesorado de geografía e historia. Objetivo de esta etapa. Enseñanza de las Ciencias Sociales: Revista de Investigación, (2), pp. 27-35.

Villalón, G. \& Pagés, B.J.(2015). Representation of indigenous people in the teaching of history in primary education in Chile: case study of texts of history of chile. Diálogo Andino, (47), pp. 27-36.

Vygotsky, L. (1986). Thought and Language, Cambridge: MIT Press.

Williamson, G. \& Hidalgo, C. (2015). Flexibilidad curricularen laimplementación de proyectos de investigación para mejorar el aprendizaje de los estudiantes. El caso de Nepso Chile. Revista Actualidades Investigativas en Educación, 15 (2), pp. 286-307.

Wineburg, S.; Martin, D. \& Monte-Sano, Ch. (2013). Reading like a historian: teaching literacy in middle y high history classrooms. Nueva York: Teachers. 